\title{
Study on Distribution Behavior in Aqueous Two-phase System about Four Kinds of Alkaloids in Coptis Chinensis
}

\author{
Fengwei Wang ${ }^{a}$, Daowu Wang ${ }^{\mathrm{b}, *}$, Qingfeng Guo ${ }^{\mathrm{c}}$ and Donghui Chen ${ }^{\mathrm{d}}$ \\ Jilin provincial Engineering Laboratory for the Complex unitization of Petro resources and Biomass, \\ Changchun University of Technology, Changchun 130012, China \\ awfwsupergirl@163.com, bwangdaowu@163.com, ${ }^{\mathrm{c}} 1072300140 @ q q . c o m,{ }^{\mathrm{d}} 187081142 @ q q . c o m$
}

Keywords: coptis chinensis alkaloids, aqueous two-phase extraction, ultraviolet spectrophotometry.

\begin{abstract}
Using aqueous two-phase to extract alkaloids in coptis chinensis. By changing the aqueous two-phase systems (molecular weight and concentration of polyethylene glycol (PEG), kinds and concentration of salt), and researched the distribution behavior of four major alkaloids in rhizoma coptidis: berberine, coptisine, palmatine and jatrorrhizin in aqueous two-phase system. Combined with ultraviolet Spectrophotometric to detect the extraction. Found that the four alkaloids extraction from high to low as follows: berberine, palmatine, jatrorrhizin and coptisine. The extraction rate of them is more than $98 \%$. Hydrophobic interaction is one of the main factors, which directly affects the extraction of aqueous two-phase system.
\end{abstract}

\section{Introduction}

The rhizome of Chinese goldthread which active ingredients are alkaloids of Coptis chinensis is rhizome of perennial herbaceous plants. The total alkaloid content is higher than $10 \%$. Four kinds of alkaloids, berberine, coptisine, palmatine and jatrorrhizine, occupy about $90 \%$ in Coptis alkaloids, of which berberine more than $50 \%{ }^{[1]}$. We use aqueous two-phase systems to extract coptis chinensis alkaloids.

Aqueous two-phase systems technique is characteristics of little loss of activity, separation of less steps, mild operation condition, easy amplification, and no organic solvent residue,isolation and purification the cell, membrane, virus, nucleic acid and other natural medicine effective component.At present, two-phase extraction is used in extraction of effective ingredients of Chinese medicine.It has also been reported for the extraction and separation of alkaloids, such as colchicine ${ }^{[2]}$, caffeine ${ }^{[3]}$ and papaverine ${ }^{[4]}$.

This experiment used aqueous two-phase extraction technique to enrichment of Rhizoma Coptidis alkaloids, which improved its medicinal value and the development and application of Coptis chinensis .At the same time, this method provided some idea for using of other traditional Chinese medicine and researching of new products.

\section{Equipment and materials}

UV1901 double beam UV spectrophotometer (Shanghai eok Instrument Co.,Ltd.), QYC-211 full incubation shaker (Shanghai Xinmiao Medical Equipment Manufacturing Co Ltd), centrifuge (Hunan Kaida Scientific Instrument Co., Ltd), analytical balance (sartorius scientific instrument (Beijing) Co., Ltd.).

Coptisine standard, berberine standard, Bamartin standard, jatrorrhizine standard were purchased from Sichuan Province Vic nilfisk Biological Technology (HPLC $\geq 98 \%$ ). Molecular weights were $600,1000,2000,6000$ of the polyethylene glycol, ammonium sulfate, potassium dihydrogen phosphate, sodium citrate were analytically pure purchased from Tianjin Municipal Guangfu fine chemical Research Institute. 


\section{Experimental method}

According to the phase diagram, add a certain amount of PEG, $\left(\mathrm{NH}_{4}\right)_{2} \mathrm{SO}_{4}$, distilled water and $3 \mathrm{~mL}$ standard drug solution (berberine, coptisine, palmatine, jatrorrhizine) to the $10 \mathrm{~mL}$ centrifuge tube with cover in a certain proportion, so that the total quality of aqueous two-phase was $8 \mathrm{~g}$. Then shake it in $200 \mathrm{rpm}$ for $2 \mathrm{~h}$ to mix the two phase fully evenly. Centrifuge in $5000 \mathrm{r} / \mathrm{min}$ for $5 \mathrm{~min}$ to accelerate the phase separation. Measure the UV absorbance by UV1901 double beam UV Spectrophotometry with aqueous solution as a reference. Measured the upper and bottom phase volume. Use a dropper to draw phase solution. Determine the content of four kinds of alkaloid in phase upper and bottom respectively ${ }^{[5]}$. The relevant formul for calculation: $R=V_{t} / V_{b}, V_{t}, V_{b}$ is the phase volume upper and bottom, respectively $(\mathrm{mL})$; distribution coefficient $\mathrm{K}=\mathrm{C}_{t} / \mathrm{C}_{\mathrm{b}}, \mathrm{C}_{t}, \mathrm{C}_{\mathrm{b}}$ is the mass concentration of Rhizoma Coptidis alkaloid, respectively $(\mathrm{mg} / \mathrm{mL})$; the mass ratio of Rhizoma Coptidis alkaloids upper and bottom is G=RK; extraction rate (\%) $Y=1 /(1+1 / R K)=1 /(1+1 / G)$

\section{Results and discussion}

Effect of salt kinds on extraction efficiencies of jatrorrhizin. Under three concentration of 12\%, $16 \%, 18 \%, 20 \%, 24 \%$ the affection of ammonium sulfate, potassium dihydrogen phosphate and sodium citrate on extraction efficiencies of jatrorrhizin is shown in Fig.1.

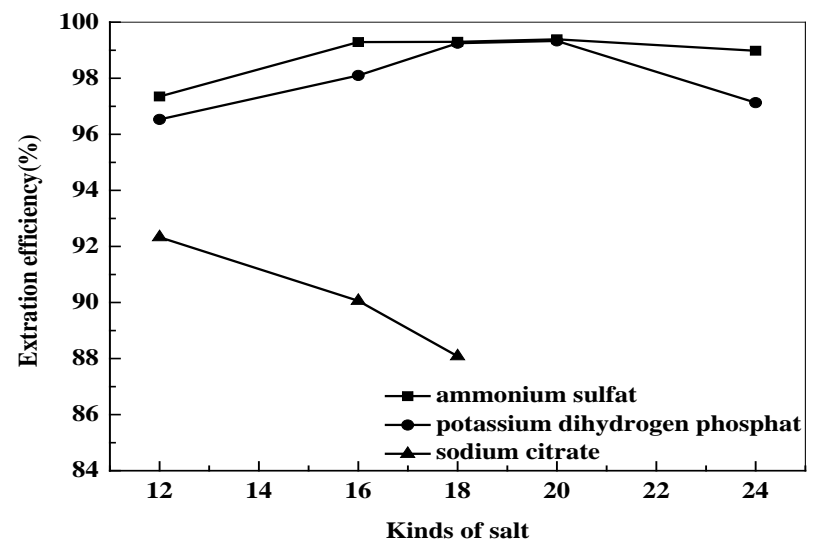

Fig. 1 Effect of salt kinds on extraction efficiencies of jatrorrhizin

Fig. 1 shows that ammonium sulfate extraction capacity is the best in five different kinds of salts. So we choose the ammonium sulfate as aqueous two-phase systems.

Phase behavior of polyethylene glycol / ammonium sulfate. According to the literature ${ }^{[5]}$ reported, the phase diagram of PEG-( $\left.\mathrm{NH}_{4}\right)_{2} \mathrm{SO}_{4}$ aqueous two-phase systems is shown in Fig.2.

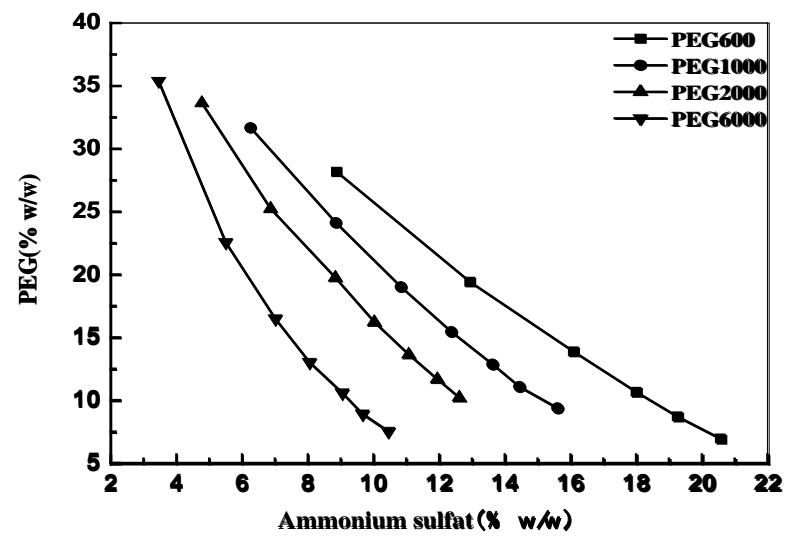

Fig.2 Phase diagram of PEG-(NH4)2SO4 aqueous two-phase systems

As shown in Figure 2, above the curve is two-phase region, while below the curve is the homogeneous phase region. Results showed that the higher the PEG molecular mass was, the lower the salt concentration was required for phase separation at the same concentration of PEG, which was 
easier to form biphasic and closer to the origin. The ability to form a two-phase order is PEG6000> PEG2000> PEG1000 > PEG600.

Meanwhile ,the viscosity of aqueous two-phase systems not only influence the separation speed and flow characteristics, but also affect the delivery of material and the distribution of particles in two phase $^{[6]}$. Because of the large molecular weight of PEG6000, the solution into a phase is too viscous, so select PEG600, PEG1000, and PEG2000 for testing.

Effect of PEG moleculer mass and concentration on extraction efficiency of berberine, berberine, palmatine, jatrorrhizine. In this experiment, when the conditions of the $\left(\mathrm{NH}_{4}\right)_{2} \mathrm{SO}_{4}$ mass fraction is $20 \%, \mathrm{pH}=6.0, \mathrm{~T}=20{ }^{\circ} \mathrm{C}$, we inspect the different molecular weight of PEG with the mass fraction of $12 \%, 16 \%, 20 \%, 24 \%, 28 \%$. The effect of PEG molecular mass and concentration on extraction efficiencies of four alkaloids is shown in Fig.3.
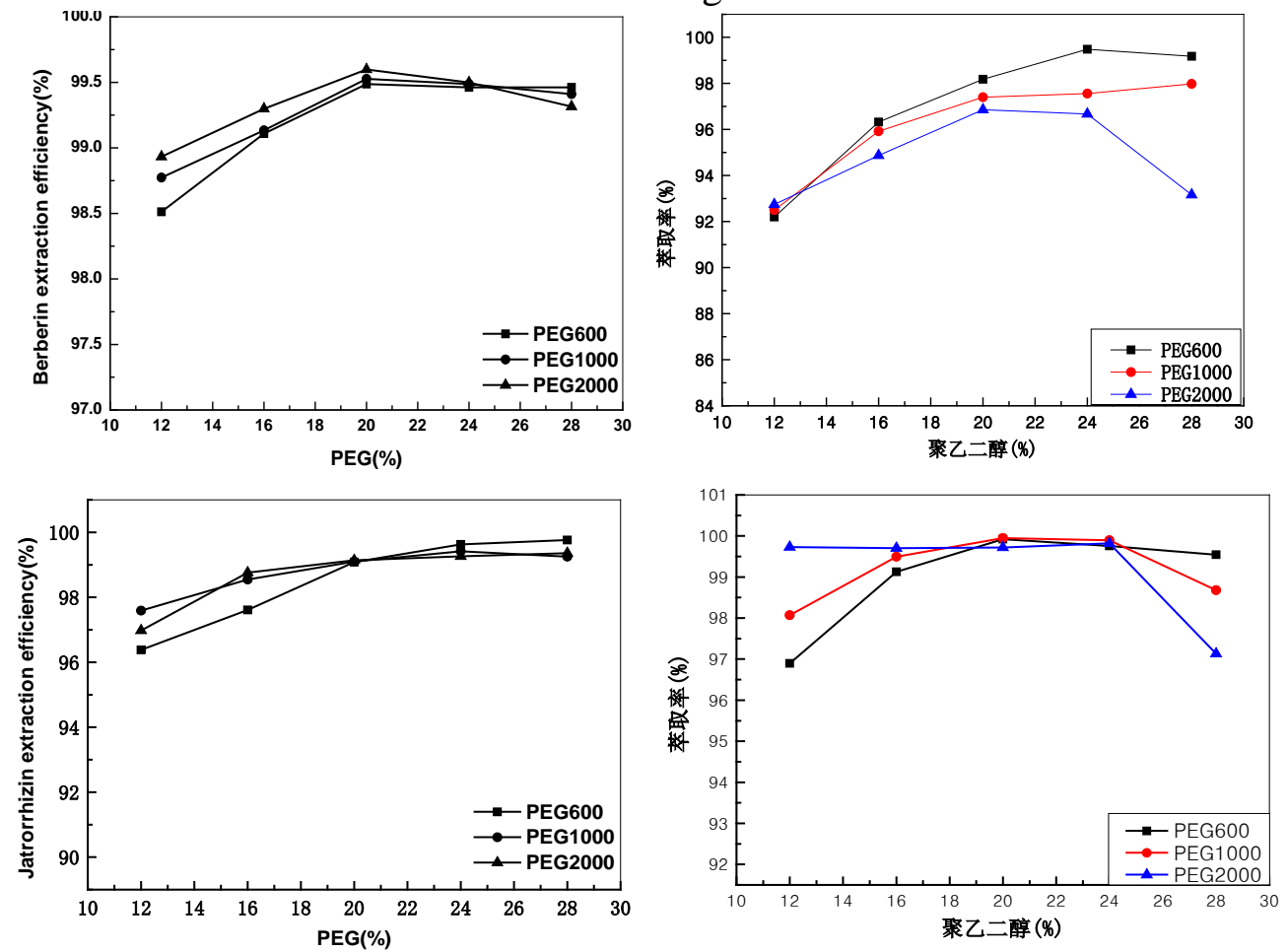

Fig.3 Effect of PEG moleculer mass and concentration on extraction efficiencies of four alkaloids

By comparing data in Fig.3, we found that the extraction rate of four alkaloids was relatively stable relative to other molecular weight, when PEG molecular weight was 1000 . So we choose PEG 1000 for testing. When the concentration of PEG was $20 \%$, berberine, palmatine has the highest extraction rate. The extraction rate of coptisine and jatrorrhizine was slightly lower than the highest values.Considering the purpose and costs of experiment, we select PEG1000 mass fraction of 20\% for testing.

Hydrophobic interactions and hydrogen bonds are two main factors, which directly affected the extraction efficiency of aqueous two-phase systems. With increasing molecular weight and concentration of PEG, the hydrophobic groups of the upper-phase including polyethylene glycol will be lager, while the extraction rate rising. When the concentration is too high, the hydrophobic chain is too large and the viscosity is too high, which influence Rhizoma Coptidis alkaloids into the upper-phase. The extraction rate decreased.

Effect of $\left(\mathrm{NH}_{4}\right)_{2} \mathrm{SO}_{4}$ concentration on extraction efficiency of berberine, berberine, palmatine, jatrorrhizine. Determining the conditions of PEG1000 mass fraction is $20 \%, \mathrm{pH}=6.0$, $\mathrm{T}=20{ }^{\circ} \mathrm{C}$, we inspect the different concentration 14\%, 16\%, 18\%, 20\%, 22\% ,24\% of $\left(\mathrm{NH}_{4}\right)_{2} \mathrm{SO}_{4}$, which impact on the extraction rate of the four alkaloids is shown in Fig. 4.

As shown in Fig.4, we found that the extraction rate of berberine, palmatine and jatrorrhizine reached the highest values, when the concentration of $\left(\mathrm{NH}_{4}\right)_{2} \mathrm{SO}_{4}$ was $16 \%$. Coptisine got the highest extraction rate in $\left(\mathrm{NH}_{4}\right)_{2} \mathrm{SO}_{4}$ concentration for $20 \%$. High salt concentration will impact on 
post-processing of aqueous two-phase system, while in order to avoid waste of raw materials, so we considered to determine the concentration of $\left(\mathrm{NH}_{4}\right)_{2} \mathrm{SO}_{4}$ is $16 \%$.

With the increase of ammonium sulfate, different distribution coefficient between positive ions and negative ions formed potential difference or interfacial tension, which can be passed to improve quality. Initially salt ions increased in the bottom phase and contended for water molecules, which led to the relative increase in polymer concentration, hydration film bigger and hydrogen bonds increased. Thus the extraction rate of alkaloids in coptis chinensis improved. When ammonium sulfate reached to a certain amount, the salt ions competed for more water molecules, instead of improving hydrophobic groups of polymers in upper-phase. The extraction rate declined.

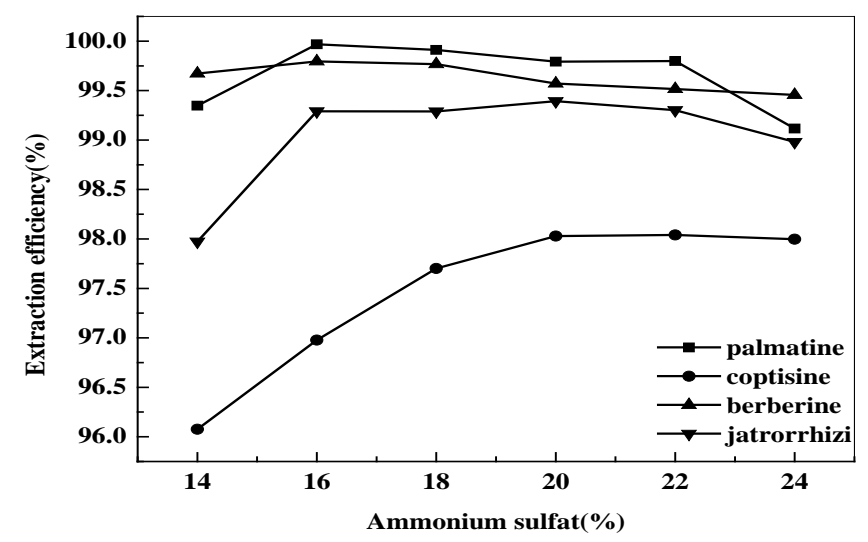

Fig.4 Effect of (NH4)2SO4 concentration on extraction efficiencies of 4 alkaloids

\section{Conclusions}

we got the best extraction conditions on rhizoma coptidis alkaloids by the experiment: $\mathrm{pH}=6, \mathrm{~T}=$ $20{ }^{\circ} \mathrm{C}$, the mass fraction of PEG1000 is $20 \%$ and the mass fraction of salt is $16 \%$. The highest extraction of berberine, coptisine, palmatine and jatrorrhizin are 99.79\%, 98.04\%, 99.96\%, 99.39\%, respectively.

Result shows that the process is simple, good stability, high extraction rate, it provides a scientific basis for the further development and utilization of rhizoma coptidis medicinal resources, and it has positive significance for the application of other Chinese herbs.

\section{References}

[1] Tang Lin.A new extraction technology of Coptis chinensis and the modern transformation for traditional preparation[D].Chongqing: Southwest University, 2011.

[2] Shi Yao, Yang yaling , Li Wanyi , Liu Mousheng . Extraction the colchicine of ipigenia indica in aqueous two-phase system [J]. Natural Product Research and Development, 2012,24: 1412- 1416.

[3] Freire M G,Neves C M S S,Marrucho I M ,et a1. High-performance extraction of alkaloids using aqueous two-phase systerns with ionic liquids [J].Green Chemistry,2010,12(10):1715-1718.

[4] Cao Qing, Li Shehong, He Chiyang, etal. Extraction and determination of papaverin in pericarpium papaveris using aqueous two-phase system of poly(ethyleneglycol)-(NH4)2SO4 coupled with high-performance liquid chromatography[J]. Analytica Chimica Acta, 2007, 590(2):187-194 .

[5] Shi Hui. Extraction and purification of flavonoids from populus canadensis moench. Leaves [D].Beijing: Beijing Forestry University, 2008.

[6] Dai Youyuan. Development and application of new extraction technologies [M]. Beijing: Chemical Industry Press, 2007. 